(2) Open Access Full Text Article

\title{
Assay development and high-throughput screening for small molecule inhibitors of a Vibrio cholerae stress response pathway
}

This article was published in the following Dove Press journal:

Drug Design, Development and Therapy

19 September 2017

Number of times this article has been viewed

\section{Laura Stanbery \\ Jyl S Matson}

Department of Medical Microbiology and Immunology, College of Medicine and Life Sciences, The University of Toledo, Toledo, OH, USA
Correspondence: Jyl S Matson

Department of Medical Microbiology and Immunology, College of Medicine and

Life Sciences, The University of Toledo, Health Science Campus, 3000 Arlington Avenue, MSI02I Toledo, OH 43614 2595, USA

Tel + I $419383397 \mid$

Fax +| 4I9383 3002

Email jyl.matson@utoledo.edu
Abstract: Antibiotics are important adjuncts to oral rehydration therapy in cholera disease management. However, due to the rapid emergence of resistance to the antibiotics used to treat cholera, therapeutic options are becoming limited. Therefore, there is a critical need to develop additional therapeutics to aid in the treatment of cholera. Previous studies showed that the extracytoplasmic stress response $\left(\sigma^{\mathrm{E}}\right)$ pathway of Vibrio cholerae is required for full virulence of the organism. The pathway is also required for bacterial growth in the presence of ethanol. Therefore, we exploited this ethanol sensitivity phenotype in order to develop a screen for inhibitors of the pathway, with the aim of also inhibiting virulence of the pathogen. Here we describe the optimization and implementation of our high-throughput screening strategy. From a primary screen of over 100,000 compounds, we have identified seven compounds that validated the growth phenotypes from the primary and counterscreens. These compounds have the potential to be developed into therapeutic agents for cholera and will also be valuable probes for uncovering basic molecular mechanisms of an important cause of diarrheal disease.

Keywords: Vibrio cholerae, stress response, $\sigma^{\mathrm{E}}$, high-throughput screening

\section{Introduction}

RpoE, encoding $\sigma^{\mathrm{E}}$, regulates a response referred to as the extracytoplasmic (or envelope) stress response in that it targets stresses that affect components of the Gram-negative cell envelope, including the periplasmic space and the outer membrane. $\sigma^{\mathrm{E}}$ is a member of the extracytoplasmic function family of alternative sigma factors, which control the expression of genes whose products function outside the bacterial cytoplasm. ${ }^{1,2} \sigma^{\mathrm{E}}$-regulated genes encode proteins that are involved in folding of proteins in the periplasm, proteases, other sigma factors, and lipopolysaccharide biogenesis and/or modification factors. . $^{3-6}$

$\sigma^{\mathrm{E}}$ has been most thoroughly studied in Escherichia coli, where it is an essential sigma factor. ${ }^{7}$ The activity of $\sigma^{\mathrm{E}}$ is negatively regulated when bacteria are not experiencing external stress. This is accomplished through the activity of RseA, a membrane-bound anti-sigma factor that binds $\sigma^{\mathrm{E}}$ and sequesters it in the inner membrane, rendering it unable to bind RNA polymerase and activate stress-inducible genes. ${ }^{8,9}$ In $E$. coli, the $\sigma^{\mathrm{E}}$ regulon is induced when misfolded outer membrane proteins (OMPs) accumulate in the periplasm (Figure 1; refer Urban ${ }^{10}$ ). A C-terminal motif of the unfolded OMPs binds to the PDZ domain of the protease DegS and induces a conformational change in the protein, which activates its protease activity. ${ }^{11}$ DegS cleaves within the RseA periplasmic domain, creating a substrate for a second protease, 


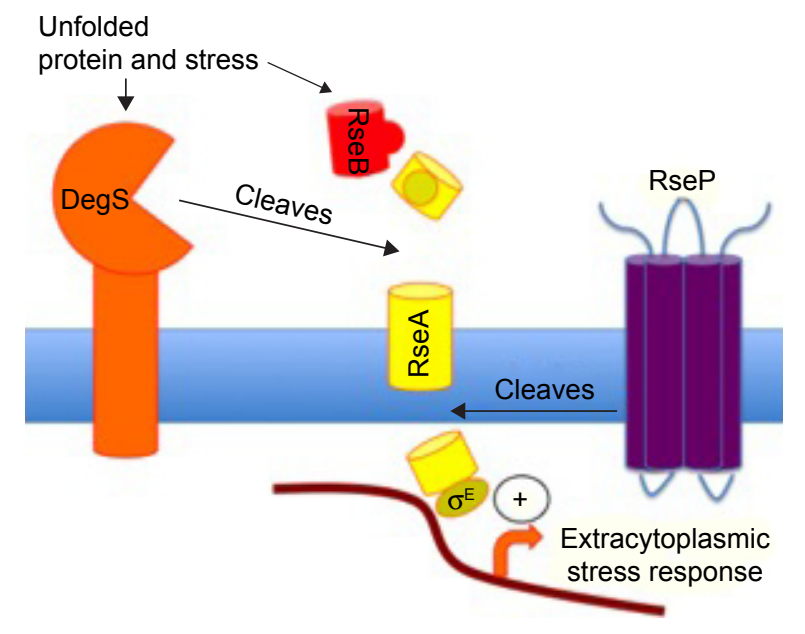

Figure I Diagram of the $\sigma^{\mathrm{E}}$ pathway.

Notes: In the absence of extracytoplasmic stress, $\sigma^{\mathrm{E}}$ is sequestered at the bacterial inner membrane through its interaction with the anti-sigma factor RseA. In the presence of unfolded outer membrane proteins or other stress, RseA is degraded in a sequential manner by the proteases DegS and RseP (also known as YaeL), releasing $\sigma^{\mathrm{E}}$ into the cytoplasm where it directs transcription of stress-inducible genes.

YaeL (RseP). YaeL then cleaves the transmembrane domain of RseA, releasing bound $\sigma^{\mathrm{E}}$ from the membrane..$^{12,13}$ The cytoplasmic ClpXP proteases further degrade RseA, releasing $\sigma^{\mathrm{E}} \cdot{ }^{14,15}$ Unbound $\sigma^{\mathrm{E}}$ complexes with core RNA polymerase at $\sigma^{\mathrm{E}}$-dependent promoters, thus activating the transcription of stress-inducible genes. ${ }^{16}$

$\sigma^{\mathrm{E}}$ plays a role in the survival of many bacterial pathogens within their hosts, making it an excellent target for potential therapeutics. For example, in Salmonella typhimurium, $\sigma^{\mathrm{E}}$ is required for protection against reactive oxygen species and antimicrobial peptides. Consequently, rpoE mutants are highly attenuated in a mouse model of infection. ${ }^{17,18}$ In Pseudomonas aeruginosa, the $\sigma^{\mathrm{E}}$ ortholog $\mathrm{AlgU}$ promotes resistance to killing by phagocytes. In addition, $\mathrm{Alg} \mathrm{U}$ is responsible for conversion of cells to the mucoid phenotype, rendering them less efficiently cleared from the respiratory tract of infected mice than nonmucoid strains. ${ }^{19,20}$ In nontypeable Haemophilus influenzae-infected macrophages, $r p o E$ is highly expressed and is required for intracellular survival. ${ }^{21}$ Mycobacterium tuberculosis rpoE mutants are defective in their ability to grow and survive inside macrophages..$^{22}$ The $\sigma^{\mathrm{E}}$ pathway is also important in Actinobacillus pleuropneumoniae and uropathogenic E. coli infection models..$^{23,24}$

$\sigma^{\mathrm{E}}$ is required for virulence in Vibrio cholerae, the causative agent of epidemic cholera. An rpoE mutant is highly attenuated in the infant mouse model of infection due to a defect in the ability to colonize the intestinal tract. ${ }^{25}$ rpoE is not required for the growth of $V$. cholerae at high temperatures, unlike E. coli and S. typhimurium. However, $r p o E$ is required for the growth and survival of classical $V$. cholerae strains in Luria-Bertani media containing 3\% ethanol. ${ }^{25}$ It is this phenotype that we targeted in developing a small molecule screen for inhibitors of the $\sigma^{\mathrm{E}}$ pathway.

Here we describe the optimization and implementation of a high-throughput screen (HTS) designed to identify inhibitors of the $\sigma^{\mathrm{E}}$ pathway. We performed the primary screen on over 100,000 small molecules, followed by counterscreening, dose-response analysis, and medicinal chemistry. We ultimately identified seven compounds that validated our initial studies. These compounds are of high interest for therapeutic development because they have the potential to inhibit this stress response and virulence in $V$. cholerae and may also target similar responses in other important bacterial pathogens. By targeting mechanisms that inhibit virulence and decrease fitness of these bacteria in vivo, such treatment strategies would be advantageous as they allow the host to clear an infection with reduced selective pressure for bacterial resistance than a traditional antibiotic.

\section{Materials and methods}

\section{Bacterial strains and culture conditions}

$V$. cholerae classical strain $\mathrm{O} 395$ was used throughout this study. The rpoE, yaeL, and $\operatorname{deg} S$ deletion strains used in this study were previously described. ${ }^{26}$ Strains were maintained at $-80^{\circ} \mathrm{C}$ in Luria-Bertani (LB) broth containing $20 \%$ glycerol. Overnight cultures were grown in $\mathrm{LB}$ medium at $37^{\circ} \mathrm{C}$ with shaking.

\section{High-throughput screening}

For the HTS assay, $30 \mu \mathrm{L}$ of $\mathrm{LB}+3 \%$ ethanol was added to each well using a Multidrop ${ }^{\mathrm{TM}}$ plate dispenser (Thermo Labsystems, Beverly, MA, USA) followed by the addition of compounds by using a Biomek ${ }^{\mathrm{TM}}$ FX Liquid Handler (Beckman $^{\circledR}$, Indianapolis, IN, USA). Ten microliters of classical $V$. cholerae strain $\mathrm{O} 395$ (in $\mathrm{LB}+3 \%$ ethanol) was then added to the sample wells by the Multidrop plate dispenser to achieve a final $\mathrm{OD}_{600}$ of $\sim 0.01$. The bacteria were added subsequent to compound addition to prevent the contamination of the liquid handler. All plates contained two columns ( 32 wells) of each control. The positive control for this assay was the rpoE deletion strain, and the negative control was the wild-type strain grown without added compound. Dimethylsulfoxide (DMSO) vehicle was added to all control wells. After 7 hours of static incubation at $37^{\circ} \mathrm{C}$, the final $\mathrm{OD}_{600}$ was read on a PHERAstar ${ }^{\circledR}$ multimode plate reader (BMG Labtech, Worcester, MA, USA). 


\section{Chemical inhibitors}

Fresh powders of 60 high priority compounds were purchased from ChemDiv (San Diego, CA, USA). Compound stock solutions were made at $20 \mathrm{mM}$ in deuterated DMSO and stored in the dark at $-20^{\circ} \mathrm{C}$. Cultures were treated with $10 \mu \mathrm{M}$ compound unless otherwise specified, as this was the concentration of compound used in the primary screen.

\section{Low-throughput screening}

Low-throughput screening of the 60 candidate compounds was performed in a volume of $1 \mathrm{~mL}$ of culture. Overnight cultures were diluted 1:100 into LB medium with and without ethanol. Compounds were initially added to cultures at the highest concentration $(20 \mu \mathrm{M})$ and then serially diluted by half to the lowest concentration tested $(2.5 \mu \mathrm{M})$ while keeping the DMSO concentration constant. Three $1 \mathrm{~mL}$ replicates of the cultures containing different concentrations of compounds (ie, $20 \mu \mathrm{M}, 10 \mu \mathrm{M}, 5 \mu \mathrm{M}$, and $2.5 \mu \mathrm{M}$ ) were grown for 7 hours with shaking at $37^{\circ} \mathrm{C}$. Control (untreated) cultures received an equal volume of DMSO. Following growth, $100 \mu \mathrm{L}$ samples of each culture replicate were placed in a 96-well plate, and the final $\mathrm{OD}_{600}$ was determined by using a CLARIOstar ${ }^{\circledR}$ plate reader (BMG Labtech).

\section{Results}

\section{Rationale for screening strategy}

Our screen for small molecule inhibitors of $\sigma^{\mathrm{E}}$ function was relatively straightforward in design: We exploited the ethanol sensitivity phenotype of $\sigma^{\mathrm{E}}$-mutant $V$. cholerae and assayed for compounds that inhibited the growth of a wildtype classical $V$. cholerae strain (O395) in the presence of 3\% ethanol. Our findings and those of others demonstrated this phenotype for an rpoE mutant (Figure 2; refer Kovacikova and Skorupski ${ }^{25}$ ). In addition, we demonstrated that mutations in the two proteases that cleave the anti-sigma factor RseA (DegS and YaeL) also showed this phenotype, likely due to constitutive sequestration of $\sigma^{\mathrm{E}}$ at the bacterial membrane in their absence (Figure 2; Matson and DiRita ${ }^{26}$ ). Importantly, no other pathways that contribute to ethanol sensitivity/ resistance have been identified in $V$. cholerae. Thus, our hypothesis was that small molecules that inhibited $\sigma^{\mathrm{E}}$ activity would cause the ethanol sensitivity phenotype. Inhibition of activity could happen at several steps in this pathway, including by interfering with the $\sigma^{\mathrm{E}}-\mathrm{RNA}$ polymerase interaction or by causing $\sigma^{\mathrm{E}}$ to be constitutively sequestered at the membrane (by inhibiting protease activity/efficiency). We reasoned that any of these steps might be inhibited by a set of compounds, resulting in numerous targets/compound

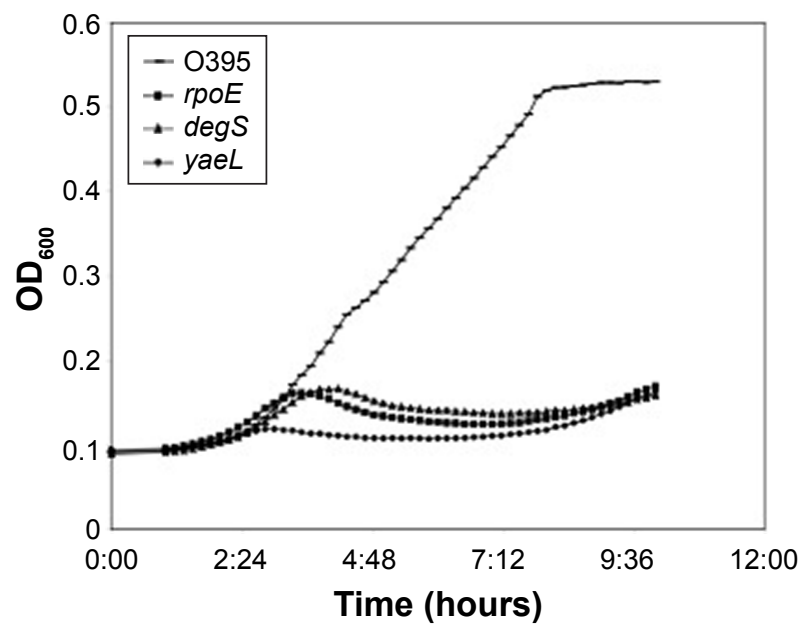

Figure $2 \sigma^{\mathrm{E}}$ pathway mutants of Vibrio cholerae cannot grow in media containing ethanol.

Notes: $\mathrm{O} 395$ (wild type) and deletion strains of $r p o E\left(\sigma^{\mathrm{E}}\right)$, degS, and yaeL were grown in 96 -well plates for 10 hours at $37^{\circ} \mathrm{C}$ with shaking in Luria-Bertani media containing $3 \%$ ethanol.

combinations that could produce the desired outcome. Therefore, we aimed to develop and optimize an HTS to identify small molecules that inhibit the growth of wild-type $V$. cholerae in the presence of $3 \%$ ethanol.

\section{Miniaturization of the assay for screening in 384-well plates}

For a screen to be considered "high-throughput," it should be optimized for the smaller-scale formats used for such assays, typically using 384- or 1,536-well microtiter plates. Since we consistently observed the growth of the wild-type strain and lack of growth for the rpoE deletion strain in 96-well plates, we did not anticipate the need for dramatic changes in the miniaturization process. For the 96-well plates, $100 \mu \mathrm{L}$ cultures of the wild type and $r p o E$ deletion strains were assayed for growth, which was scaled down to $40 \mu \mathrm{L}$ cultures in the 384 -well format. We initially tested the growth phenotypes of the two strains in 384-well plates in the presence of $3 \%$ ethanol and read the $\mathrm{OD}_{600}$ of the cultures after either 7 hours or overnight growth at $37^{\circ} \mathrm{C}$ The plates were incubated without shaking, in contrast to previous assays, as that would be optimal for subsequent HTS. Although the final $\mathrm{OD}_{600}$ of the wild-type cells was lower in this smaller format, there was still a clear difference between the wild-type strain, which grew to an $\mathrm{OD}_{600}$ of $\sim 0.18$ at 7 hours, and the $r p o E$ mutant, which remained at the level of detection ( 0.05). After overnight growth, there was no longer a significant difference in $\mathrm{OD}_{600}$ between strains. Therefore, we proceeded with the optimization of the assay at the 7-hour time point. 
A key parameter for determining whether an assay is robust enough to use in a large-scale screen is the $Z$ ' factor. ${ }^{27}$ This statistical parameter is calculated to evaluate the effectiveness of an assay used for HTS. An assay with a very large dynamic range (difference in average signal between the positive and negative controls) and a very small SD has a Z' factor approaching the theoretically perfect value of 1 . An assay with a $Z$ ' factor between 0.5 and 1 is considered an excellent assay; therefore, we sought to optimize our assay to fall within this range before proceeding to screening compounds. After several preliminary experiments, through which we determined that sealing the plates in plastic is necessary to prevent the evaporation of media in wells near the edges of the plates, we obtained a reliable $Z$ ' factor $>0.7$ for this assay (Figure 3). Therefore, we concluded that the assay was robust enough in the 384-well format to proceed with a small-scale pilot screen.

\section{Pilot screen}

A screen of the 2,000 compounds of Spectrum Library and the 446 compounds of National Institutes of Health (NIH) clinical collection was carried out at the Center for Chemical Genomics (CCG) in the Life Sciences Institute at the University of Michigan. The Spectrum Collection from MicroSource Discovery Systems, Inc. consists of 70 compounds $(3.5 \%)$ approved for and restricted to

$\mathbf{0 3 9 5}$ (wt)

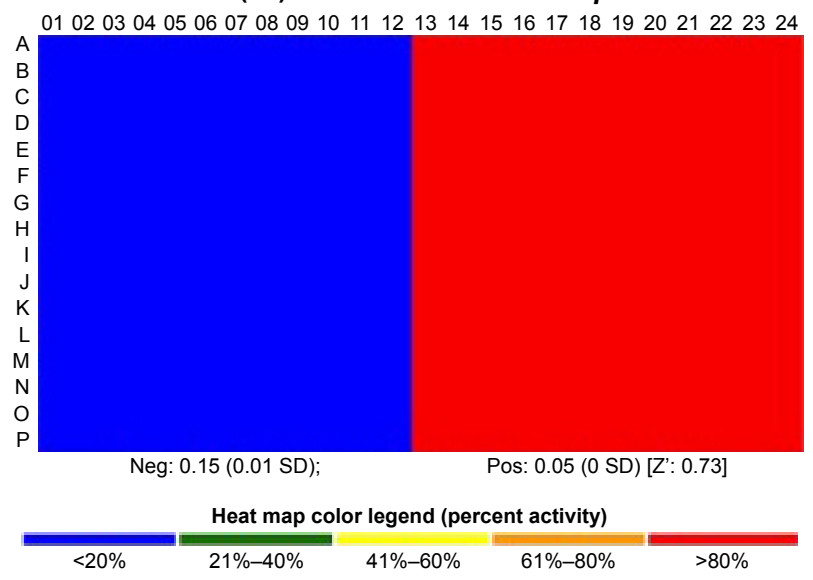

Figure 3 Wild-type Vibrio cholerae and an rpoE deletion strain grow to significantly different densities in the 384-well format.

Notes: The wells in the left half of a 384-well plate were inoculated with 0395 (ethanol-sensitive negative control), and the wells in the right half were inoculated with the rpoE deletion strain (ethanol-sensitive positive control) in media containing $3 \%$ ethanol and DMSO vehicle control. The plate was sealed in a plastic bag and incubated at $37^{\circ} \mathrm{C}$ for 7 hours, when the $O D_{600}$ was recorded. $\mathrm{O} 395(\mathrm{Neg})$ grew to an average OD of 0.15 and the rpoE deletion strain (Pos) grew to an average OD of 0.05 . The Z' factor for this individual experiment was 0.73 .

Abbreviations: DMSO, dimethylsulfoxide; Neg, negative; Pos, positive; wt, wild type. agricultural use, 343 compounds (17.15\%) with reported biological activities at the experimental level, 629 natural products and derivatives (31.45\%) with undetermined biological activities, and 958 known drugs (47.9\%) that have been used in human therapy. The NIH clinical collection consists of 446 compounds that have a history of use in human clinical trials. This screen was accomplished in the 384-well format as described in the "Materials and methods" section. The assay performed well in this pilot assay $\left(Z^{\prime}=0.76\right.$, signal $/$ noise $=3$, coefficient of variation $[\%]=8.3$ [negative control]) as illustrated in Figure 4. Based on the commonly used cutoff of three standard deviations from the mean (Figure 4, red line), 41 compounds $(1.7 \%)$ answered our pilot screen and would be considered "actives." Due to the success of this pilot assay, we concluded that the screen was suitable for proceeding with HTS of a larger library.

\section{Large-scale HTS for candidate $\sigma^{\mathrm{E}}$ inhibitors}

The optimized primary screen was used to test an additional 99,680 diverse compounds from the ChemDiv $100 \mathrm{~K}$ collection at the CCG screening facility. This brought the total number of compounds screened to 102,126 including those from the pilot screen. Compounds were used at a concentration of $10 \mu \mathrm{M}$ for primary screening. Compounds were initially assessed for their ability to inhibit growth in the presence of $3 \%$ ethanol, similar to the rpoE mutant (the positive control for this screen). After primary screening, we removed all compounds showing $\geq 45 \%$ of the positive control value (Figure 5). The remaining compounds were confirmed by repeating the assay in triplicate. Any compounds that did not inhibit growth to this cutoff in three of the four repetitions were excluded from further study. At this stage, we also tested the effect of these compounds on $V$. cholerae growth in the absence of ethanol to exclude those that generally inhibited bacterial growth, such as antibiotics. This counterscreen was included to increase the likelihood that we would identify compounds that targeted the $\sigma^{\mathrm{E}}$ pathway and were not nonspecific inhibitors of $V$. cholerae replication. Compounds that inhibited growth in the absence of ethanol $(>50 \%)$ were removed from further study.

Compounds remaining in the pool at this stage were subsequently tested for their dose-response characteristics. Assays were carried out using a range of concentrations of compound from 1 to $100 \mu \mathrm{M}$ to determine the concentration required to inhibit the assay to $50 \%$ of its maximum value $\left(\mathrm{IC}_{50}\right)$. Compounds that did not exhibit a good relationship 


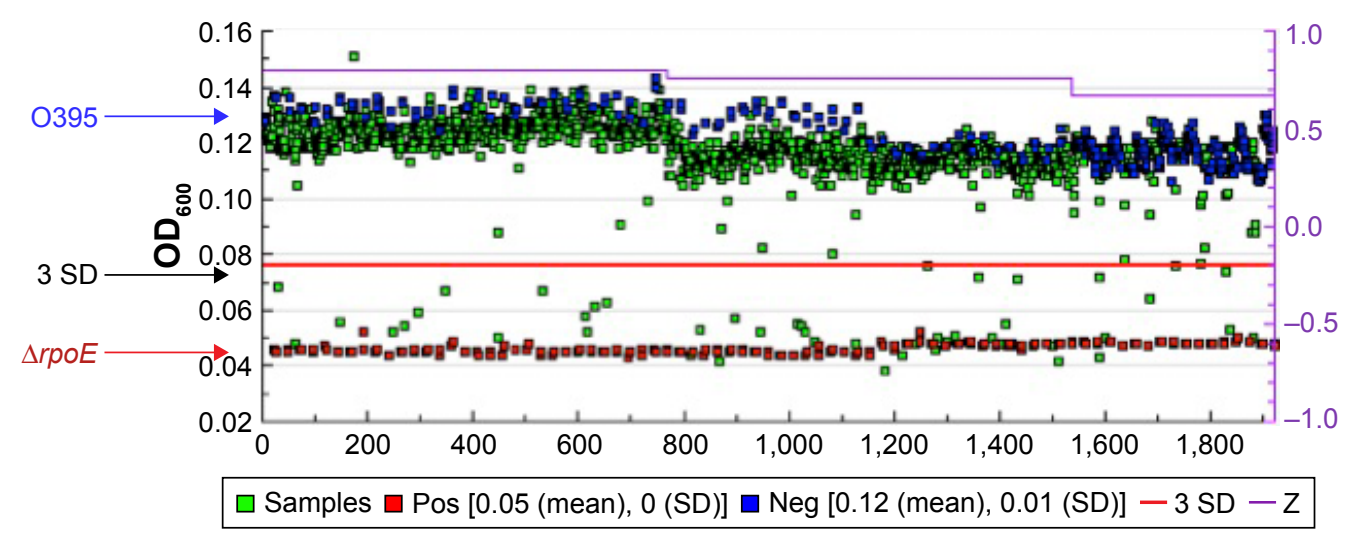

Figure 4 Pilot screen for small molecule inhibitors of Vibrio cholerae growth in the presence of $3 \%$ ethanol.

Notes: Bacteria were seeded into wells containing compound to a final $\mathrm{OD}_{600}$ of $\sim 0.0 \mathrm{I}$. Two columns of each control strain with DMSO vehicle were included as controls for each plate. O395 served as the ethanol-sensitive negative control ( $\mathrm{Neg}$, blue), and the rpoE mutant served as the ethanol-sensitive positive control (Pos, red) for the purpose of this screen. The rest of the plate contained individual compounds in each well. Growth levels in experimental wells are represented as green data points (Samples). The plates were sealed in plastic bags and incubated at $37^{\circ} \mathrm{C}$ for 7 hours when the $\mathrm{OD}_{600}$ was recorded. Representative view of final $\mathrm{OD}_{600}$ readings is from 384 -well plates.

Abbreviations: DMSO, dimethylsulfoxide; Neg, negative; Pos, positive.

between the concentration and the level of inhibition were excluded from further study. The remaining compounds were then prioritized for further study by a medicinal chemist based on potency, potential for toxicity/reactivity, and so on. By applying medicinal chemistry analysis, the compounds were categorized into high, medium, and low priority groups for further study. The next step was to establish confidence in the activity of any compounds we were interested in pursuing further. To that end, we purchased 60 of the highest priority compounds from ChemDiv in order to confirm the activity observed in the primary assay using fresh powders.

\section{Compound validation}

The purchased compounds were retested for growth inhibition in the presence of ethanol in a low-throughput format. Seven of the 60 compounds demonstrated reduced growth ( $\leq 50 \%$ of untreated cultures at $10 \mu \mathrm{M}$ ), validating our previous studies (Figure 6). In addition, all compounds were

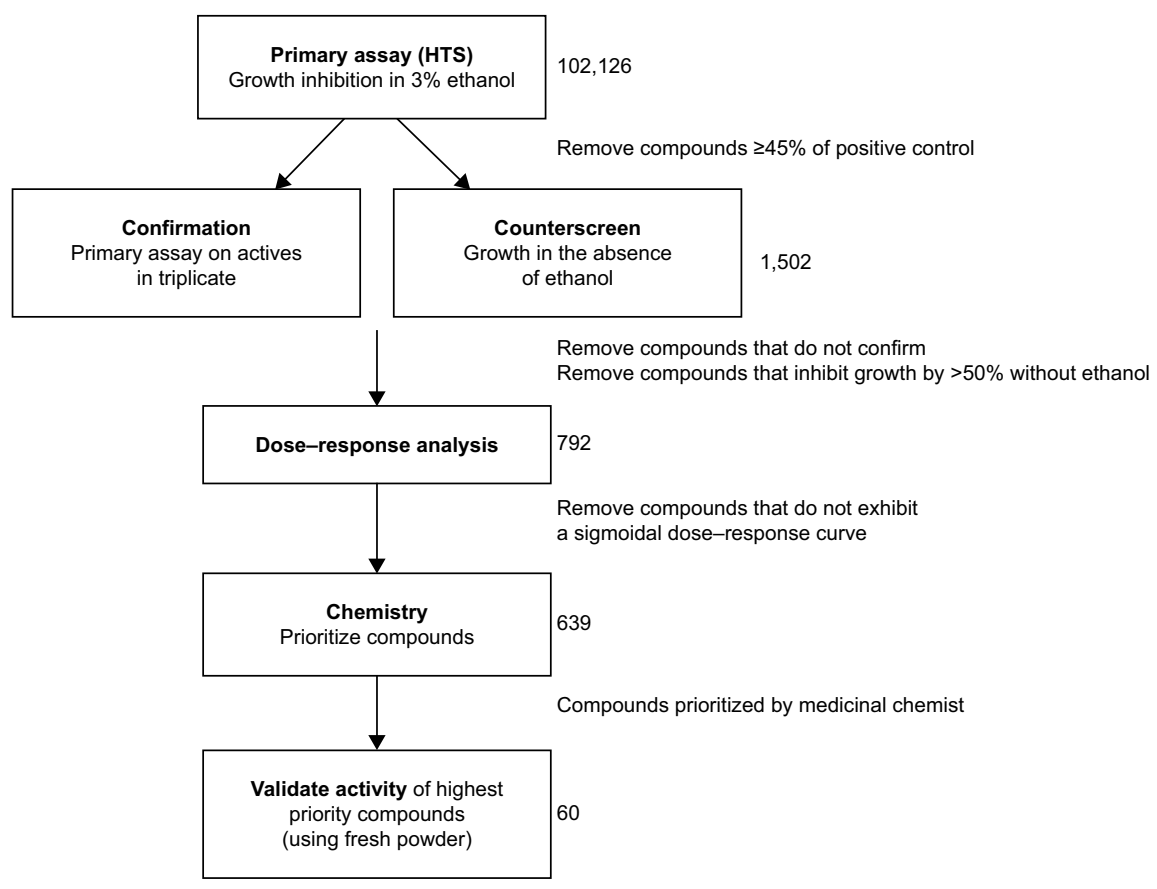

Figure 5 Flowchart of screening and triage strategy.

Notes: Criteria for including/excluding compounds at each step are indicated, as well as the number of remaining compounds.

Abbreviation: HTS, high-throughput screen. 


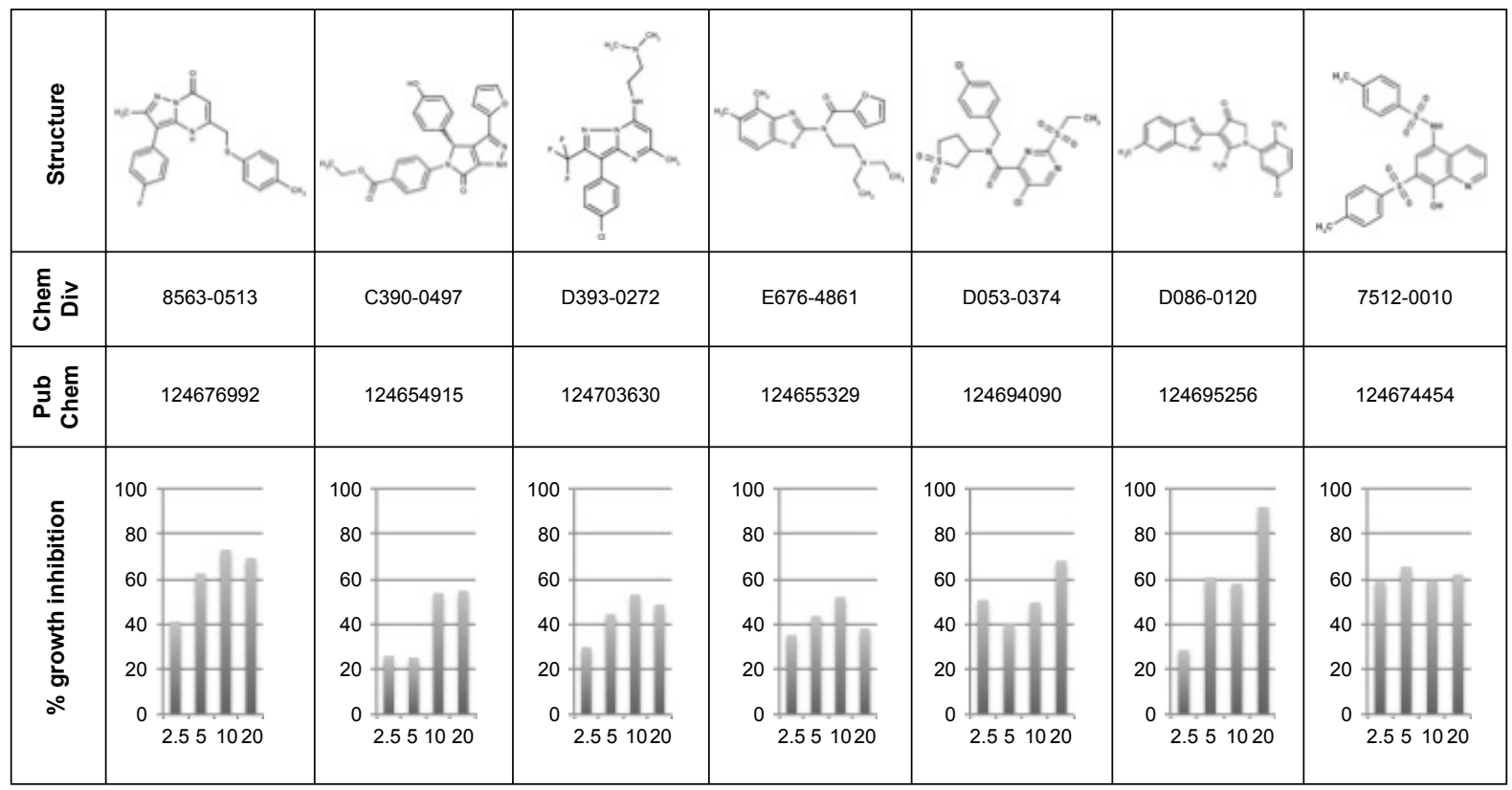

Figure 6 Lead compounds.

Notes: Of the 60 compounds purchased from ChemDiv for retesting, seven showed reduced growth in the presence of ethanol and limited toxicity in its absence. For each of the seven compounds, the chemical structure, ChemDiv ID, PubChem ID, and percent growth inhibition (relative to untreated cultures) are shown at $2.5 \mu \mathrm{M}, 5 \mu \mathrm{M}$, $10 \mu \mathrm{M}$, and $20 \mu \mathrm{M}$.

tested for growth inhibition in the absence of ethanol, as was previously performed as a counterscreen. None of these seven compounds significantly reduced growth at a concentration of $10 \mu \mathrm{M}$, although some showed toxicity at higher concentrations (Figure S1). We consider these seven compounds to be our lead compounds for mechanistic studies and potential therapeutic development. The structures of the seven compounds do not appear to be closely related, which may indicate that different steps in the $\sigma^{\mathrm{E}}$ pathway are targeted by different compounds or that some of the compounds targeted an unidentified pathway that results in the same growth phenotype. Future studies aim to characterize the mechanism of action of each of the lead compounds and to determine their ability to reduce $V$. cholerae virulence in vivo.

\section{Discussion}

In spite of the use of oral rehydration therapy (ORT), antibiotics, and recently developed vaccines, cholera remains a significant cause of morbidity and mortality in many parts of the world..$^{28}$ ORT is the first line of treatment, replacing the fluids lost by the patient and allowing the infection to run its course. ${ }^{29}$ ORT is occasionally supplemented by antibiotic treatment, which reduces the symptoms/duration of disease and limits spread to contacts. ${ }^{30-32}$ However, as with most bacterial diseases, antibiotic resistance in $V$. cholerae is increasing rapidly. Therefore, we and others are actively pursuing alternative therapeutic options to improve the treatment of cholera infections. ${ }^{33-35}$

In this study, we used a cell-based, HTS to target a conserved Gram-negative stress response pathway. Although we ultimately identified seven compounds that validated the expected phenotype after purchase and reexamination, most of the purchased compounds did not behave as would be expected. Upon testing the newly purchased compounds, we identified those that did not show any growth inhibition in the presence of ethanol, those that showed significant growth inhibition (toxicity) in the absence of ethanol, and those that inhibited growth in the presence of ethanol, but not at a significant level that would make them candidates for further study. This lack of reproducibility is likely due to the gradual degradation of some compounds in the original screening library. The originally observed phenotypes may have been due to a breakdown product and not the original small molecule. This is why it is critical to validate any phenotypes of interest using freshly purchased compounds.

The strategy of inhibiting a well-characterized stress response mechanism is advantageous for several reasons. Targeting such a pathway is ideal as it does not inhibit virulence per se, but alters a pathway in such a way that treatment with these compounds would decrease the fitness of the organism in the host. Inhibition of bacterial fitness is one of many strategies that are currently being explored 
in response to increasing antibiotic resistance in bacterial pathogens. ${ }^{36}$ When fitness of a pathogen is decreased in the human host, spread or growth of the organism may be limited, allowing the host microbiota to outcompete the pathogen. In addition, when a therapeutic agent is used that does not kill the pathogen, it is less likely to select for resistance as the selection pressure is less intense. Identification of fitness factors in $V$. cholerae and a range of other bacterial pathogens is underway, due to their potential for therapeutic development. ${ }^{37-39}$

An additional benefit of targeting this specific pathway is the fact that $\sigma^{\mathrm{E}}$ plays a role in the survival of many other important bacterial pathogens within their hosts. Therefore, it is expected that inhibitors of this conserved pathway could be developed into treatments for other bacterial infections. In addition to the therapeutic potential of such compounds, small molecules have significant value as molecular probes for studying the basic biology of bacterial pathogens. Because these compounds do not inhibit growth or kill the bacteria in the absence of ethanol, they can be used experimentally to study this stress response pathway without genetically altering the pathogen. Due to the conserved nature of this pathway, such probes may be of particular use for studying the $\sigma^{\mathrm{E}}$ pathway in bacteria where some of these stress response genes are essential for growth and survival.

\section{Conclusion}

We have designed and successfully carried out a novel HTS targeting a stress response pathway that plays a role in the virulence of $V$. cholerae. In addition, we provide confirmatory and counterassays, allowing us to ultimately identify seven lead compounds that are of significant interest for future therapeutic development. Current work focuses on better characterizing the mechanism of action of each of the seven lead compounds, ie, validating that the $\sigma^{\mathrm{E}}$ pathway is the target of each compound and subsequently determining what step in the $\sigma^{\mathrm{E}}$ pathway is impacted. Our future goals are to test lead compounds for their toxicity in cultured human cells and then to test any nontoxic compounds for their ability to reduce colonization in an animal model of $V$. cholerae infection.

\section{Acknowledgments}

We would like to thank the CCG in the University of Michigan - specifically Martha Larsen, Steve Vander Roest, and Hollis Showalter - for assistance with the small molecule screening. The authors would also like to thank Victor DiRita for helpful discussions and critical reading of the manuscript. Funding for these studies was provided by the Michigan Institute for Clinical and Health Research Pilot Grant Program Seed Grant 2UL1TR000433 from the University of Michigan and NICHD HD075928 from the NIH.

\section{Disclosure}

The authors report no conflicts of interest in this work.

\section{References}

1. Helmann JD. The extracytoplasmic function (ECF) sigma factors Adv Microb Physiol. 2002;46:47-110.

2. Ruiz N, Kahne D, Silhavy TJ. Advances in understanding bacterial outer-membrane biogenesis. Nat Rev Microbiol. 2006;4(1):57-66.

3. Alba BM, Gross CA. Regulation of the Escherichia coli sigma-dependent envelope stress response. Mol Microbiol. 2004;52(3):613-619.

4. Dartigalongue C, Missiakas D, Raina S. Characterization of the Escherichia coli sigma E regulon. J Biol Chem. 2001;276(24):20866-20875.

5. Rezuchova B, Miticka H, Homerova D, Roberts M, Kormanec J. New members of the Escherichia coli sigmaE regulon identified by a twoplasmid system. FEMS Microbiol Lett. 2003;225(1):1-7.

6. Rhodius VA, Suh WC, Nonaka G, West J, Gross CA. Conserved and variable functions of the sigmaE stress response in related genomes. PLoS Biol. 2006;4(1):e2.

7. De Las Penas A, Connolly L, Gross CA. SigmaE is an essential sigma factor in Escherichia coli. J Bacteriol. 1997;179(21):6862-6864.

8. De Las Penas A, Connolly L, Gross CA. The sigmaE-mediated response to extracytoplasmic stress in Escherichia coli is transduced by RseA and RseB, two negative regulators of sigmaE. Mol Microbiol. 1997; 24(2):373-385.

9. Missiakas D, Mayer MP, Lemaire M, Georgopoulos C, Raina S. Modulation of the Escherichia coli sigmaE (RpoE) heat-shock transcriptionfactor activity by the RseA, RseB and RseC proteins. Mol Microbiol. 1997;24(2):355-371.

10. Urban S. Making the cut: central roles of intramembrane proteolysis in pathogenic microorganisms. Nature Rev Microbiol. 2009;7(6): 411-423.

11. Walsh NP, Alba BM, Bose B, Gross CA, Sauer RT. OMP peptide signals initiate the envelope-stress response by activating DegS protease via relief of inhibition mediated by its PDZ domain. Cell. 2003; 113(1):61-71.

12. Alba BM, Leeds JA, Onufryk C, Lu CZ, Gross CA. DegS and YaeL participate sequentially in the cleavage of RseA to activate the sigma(E)dependent extracytoplasmic stress response. Genes Dev. 2002;16(16): 2156-2168.

13. Kanehara K, Ito K, Akiyama Y. YaeL (EcfE) activates the sigma(E) pathway of stress response through a site-2 cleavage of anti-sigma(E), RseA. Genes Dev. 2002;16(16):2147-2155.

14. Flynn JM, Levchenko I, Sauer RT, Baker TA. Modulating substrate choice: the SspB adaptor delivers a regulator of the extracytoplasmicstress response to the AAA+ protease ClpXP for degradation. Genes Dev. 2004;18(18):2292-2301.

15. Flynn JM, Neher SB, Kim YI, Sauer RT, Baker TA. Proteomic discovery of cellular substrates of the ClpXP protease reveals five classes of ClpX-recognition signals. Mol Cell. 2003;11(3):671-683.

16. Chaba R, Grigorova IL, Flynn JM, Baker TA, Gross CA. Design principles of the proteolytic cascade governing the sigmaE-mediated envelope stress response in Escherichia coli: keys to graded, buffered, and rapid signal transduction. Genes Dev. 2007;21(1):124-136.

17. Humphreys S, Stevenson A, Bacon A, Weinhardt AB, Roberts M. The alternative sigma factor, sigmaE, is critically important for the virulence of Salmonella typhimurium. Infect Immun. 1999;67(4): $1560-1568$. 
18. Testerman TL, Vazquez-Torres A, Xu Y, Jones-Carson J, Libby SJ, Fang FC. The alternative sigma factor sigmaE controls antioxidant defences required for Salmonella virulence and stationary-phase survival. Mol Microbiol. 2002;43(3):771-782.

19. Boucher JC, Schurr MJ, Yu H, Rowen DW, Deretic V. Pseudomonas aeruginosa in cystic fibrosis: role of mucC in the regulation of alginate production and stress sensitivity. Microbiology. 1997;143(Pt 11): 3473-3480.

20. Deretic V, Schurr MJ, Boucher JC, Martin DW. Conversion of Pseudomonas aeruginosa to mucoidy in cystic fibrosis: environmental stress and regulation of bacterial virulence by alternative sigma factors. J Bacteriol. 1994;176(10):2773-2780.

21. Craig JE, Nobbs A, High NJ. The extracytoplasmic sigma factor, final sigma(E), is required for intracellular survival of nontypeable Haemophilus influenzae in $\mathbf{J} 774$ macrophages. Infect Immun. 2002; 70(2):708-715.

22. Manganelli R, Voskuil MI, Schoolnik GK, Smith I. The Mycobacterium tuberculosis ECF sigma factor sigmaE: role in global gene expression and survival in macrophages. Mol Microbiol. 2001;41(2):423-437.

23. Redford P, Roesch PL, Welch RA. DegS is necessary for virulence and is among extraintestinal Escherichia coli genes induced in murine peritonitis. Infect Immun. 2003;71(6):3088-3096.

24. Sheehan BJ, Bosse JT, Beddek AJ, Rycroft AN, Kroll JS, Langford PR. Identification of Actinobacillus pleuropneumoniae genes important for survival during infection in its natural host. Infect Immun. 2003; 71(7):3960-3970.

25. Kovacikova G, Skorupski K. The alternative sigma factor sigma(E) plays an important role in intestinal survival and virulence in Vibrio cholerae. Infect Immun. 2002;70(10):5355-5362.

26. Matson JS, DiRita VJ. Degradation of the membrane-localized virulence activator TcpP by the YaeL protease in Vibrio cholerae. Proc Natl Acad Sci US A. 2005;102(45):16403-16408.

27. Zhang JH, Chung TD, Oldenburg KR. A simple statistical parameter for use in evaluation and validation of high throughput screening assays. J Biomol Screen. 1999;4(2):67-73.
28. Qadri F, Wierzba TF, Ali M, et al. Efficacy of a single-dose, inactivated oral cholera vaccine in Bangladesh. $N$ Engl J Med. 2016;374(18): 1723-1732.

29. Guerrant RL, Carneiro-Filho BA, Dillingham RA. Cholera, diarrhea, and oral rehydration therapy: triumph and indictment. Clin Infect Dis. 2003;37(3):398-405.

30. Greenough WB 3rd, Gordon RS Jr, Rosenberg IS, Davies BI, Benenson AS. Tetracycline in the treatment of cholera. Lancet. 1964; 1(7329):355-357.

31. Lindenbaum J, Greenough WB, Islam MR. Antibiotic therapy of cholera in children. Bull World Health Organ. 1967;37(4):529-538.

32. Nelson EJ, Nelson DS, Salam MA, Sack DA. Antibiotics for both moderate and severe cholera. N Engl J Med. 2011;364(1):5-7.

33. Fazil MH, Singh DV. Vibrio cholerae infection, novel drug targets and phage therapy. Future Microbiol. 2011;6(10):1199-1208.

34. Hung DT, Shakhnovich EA, Pierson E, Mekalanos JJ. Small-molecule inhibitor of Vibrio cholerae virulence and intestinal colonization. Science. 2005;310(5748):670-674.

35. Yamasaki S, Asakura M, Neogi SB, Hinenoya A, Iwaoka E, Aoki S. Inhibition of virulence potential of Vibrio cholerae by natural compounds. Indian J Med Res. 2011;133:232-239.

36. National Research Council. Treating Infectious Diseases in a Microbial World: report of Two Workshops on Novel Antimicrobial Therapeutics; Washington, DC: The National Academies Press; 2006.

37. Bachman MA, Breen P, Deornellas V, et al. Genome-wide identification of Klebsiella pneumoniae fitness genes during lung infection. MBio. 2015;6(3):e00775.

38. Johnson CN. Fitness factors in vibrios: a mini-review. Microb Ecol. 2013;65(4):826-851.

39. Subashchandrabose S, Mobley HL. Virulence and fitness determinants of uropathogenic Escherichia coli. Microbiol Spectr. 2015;3(4). 


\section{Supplementary material}
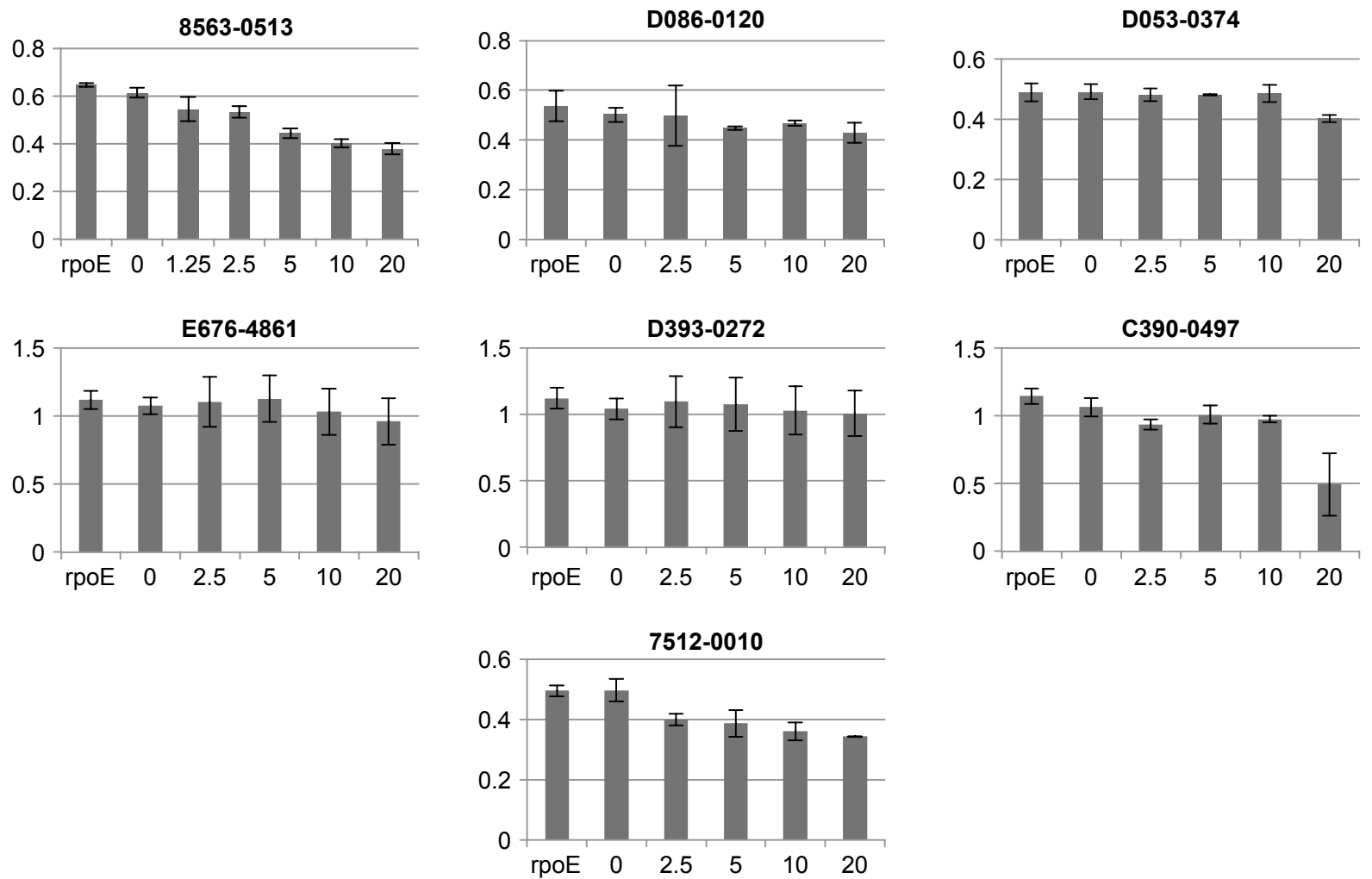

Figure SI Vibrio cholerae growth in the presence of lead compounds.

Notes: The seven lead compounds were assayed for growth inhibition of $V$. cholerae in the absence of ethanol to assess general toxicity of the compounds. The OD 600 of the rpoE mutant (rpoE; positive control) and $\mathrm{O} 395$ (wt) grown in the presence of increasing concentrations of compound $(\mu \mathrm{M})$ are shown.

\section{Publish your work in this journal}

Drug Design, Development and Therapy is an international, peerreviewed open-access journal that spans the spectrum of drug design and development through to clinical applications. Clinical outcomes, patient safety, and programs for the development and effective, safe, and sustained use of medicines are the features of the journal, which has also been accepted for indexing on PubMed Central. The manuscript management system is completely online and includes a very quick and fair peer-review system, which is all easy to use. Visit http://www.dovepress.com/testimonials.php to read real quotes from published authors. 